\title{
Co-infection of Borrelia burgdorferi sensu lato and Rickettsia species in ticks and in an erythema migrans patient
}

\author{
Ellen Tijsse-Klasen ${ }^{1 *}$, Hein Sprong ${ }^{1}$ and Nenad Pandak ${ }^{2}$
}

\begin{abstract}
Background: Lyme borreliosis is the most prevalent tick-borne disease in Europe. Ixodes ricinus also carries other pathogenic bacteria, but corresponding human diseases are rarely reported. Here, we compared the exposure to Rickettsia helvetica and Rickettsia monacensis with that to Lyme borreliosis spirochetes. We assumed that their exposure corresponds to their infection rate in questing I. ricinus.
\end{abstract}

Findings: Three Rickettsia species were detected in ticks with a total prevalence of $7.9 \%$, of which the majority was R. helvetica (78\%) and R. monacensis (21\%). From the same geographic area, skin biopsies of erythema migrans patients were investigated for possible co-infections with Rickettsia spp.. Forty-seven out of 67 skin biopsies were PCR positive for Borrelia burgdorferi s.l. and one sample was positive for R. monacensis. The Borrelia genospecies from the $R$. monacensis positive patient was identified as Borrelia afzelii. The patient did not show any symptoms associated with rickettsiosis.

Conclusions: Co-infections of I. ricinus with Rickettsia spp. and B. burgdorferi s.l. were as high as expected from the individual prevalence of both pathogens. Co-infection rate in erythema migrans patients corresponded well with tick infection rates. To our knowledge, this is the first reported co-infection of B. afzelii and R. monacensis.

Keywords: Borrelia burgdorferi, Rickettsia monacensis, Rickettsia helvetica, Erythema migrans, Co-infection

\section{Findings}

Erythema migrans (EM) is an early sign of Lyme disease, which is caused by Borrelia burgdorferi sensu lato (s.l.) spirochetes that are transmitted by ticks. A typical EM is characterized by a bull's-eye look with an expanding red ring of active infection and inner clearing but other presentations of EM can be found as well [1]. As a first sign of Lyme disease, it usually occurs at the site of the tick bite and is associated with tick attachment duration of more than 24 hours [2]. These premises make EM likely sites of co-infection with other tick borne pathogens that can be transmitted alongside $B$. burgdorferi s.l.. Rickettsia spp. are, next to B. burgdorferi s.l., the most common potential pathogens found in Ixodes ricinus, the main

\footnotetext{
* Correspondence: Ellen.TijsseKlasen@gmail.com

${ }^{1}$ Centre for Infectious Disease Control Netherlands, National Institute for

Public Health and Environment (RIVM), Bilthoven, The Netherlands

Full list of author information is available at the end of the article
}

vector of Lyme disease in Europe [3-6]. Some Rickettsia species are well-established pathogens, while the pathogenic potential of others has not been fully elucidated [7]. Invasion and potentially infecting tissue at the tick-bite site is the first step tick-borne bacteria have to make in order to cause disease. We investigated the co-infection rates of ticks with these two pathogens and co-infections in EM patients from Croatia. Part of the data from this study has been published in a separate manuscript dealing with focus on the genetic variation of Borrelia genotypes [8].

For this purpose I. ricinus and EM skin biopsies were tested with a B. burgdorferi s.l. duplex qPCR and a conventional Rickettsia spp. PCR. DNA from vegetation ticks and skin biopsies of EM patients was extracted and tested for the presence of B. burgdorferi s.l. DNA [8]. Samples were tested for Rickettsia by conventional PCR

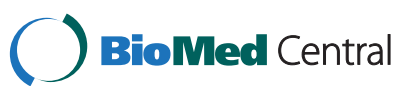

(c) 2013 Tijsse-Klasen et al.; licensee BioMed Central Ltd. This is an open access article distributed under the terms of the Creative Commons Attribution License (http://creativecommons.org/licenses/by/2.0), which permits unrestricted use, distribution, and reproduction in any medium, provided the original work is properly cited. 
on the 16S rRNA gene and PCR-positive samples were sequenced and analyzed as described [4,9]. Positive and negative controls were run with each tested batch. Positive control for the Rickettsia spp. PCR was a Rickettsia africae positive patient sample [10]. Rickettsia africae is not found in I. ricinus and can be differentiated from rickettsial species in I. ricinus based on the $16 \mathrm{~S}$ rRNA gene.

\section{Prevalence of Borrelia burgdorferi s.l. and Rickettsia spp. in} Croatian ticks and EM skin biopsies

Of 1432 I. ricinus tested for Borrelia 254 (17.7\%) were positive. Of 1273 I. ricinus tested for Rickettsia spp. 101 (7.9\%) were positive [8]. Of these, 79 (78\%) were identified as Rickettsia helvetica (100\% homology to L36212), $21(21 \%)$ as Rickettsia monacensis (100\% homology to [GenBank:DQ100164]) and one (1\%) as Rickettsia raoultii (100\% homology to [GenBank:DQ365809]). Of 67 EM skin samples 47 were B. burgdorferi s.l. positive (70\%) and one (1.5\%) was Rickettsia sp. positive. The latter sample was also positive for $B$. afzelii and the rickettsial species was identified as $R$. monacensis (100\% homology to [GenBank:DQ365809]).

\section{Case description}

The $R$. monacensis positive patient was an eight-year-old girl living in an urban environment. Six days prior to the hospital visit the patients mother had noticed a red annular rash on her daughter's face. The rash expanded throughout the following day and during the hospital visit three EM lesions were noticed: around the left eye $(7 \times 14 \mathrm{~cm})$, at the anterior side of the right thigh $(9 \times 12 \mathrm{~cm})$ and on the right gluteus $(11 \times 17 \mathrm{~cm})$. The skin biopsy was taken from the gluteal EM. The patient complained about itching but no other symptoms were reported or noted during the visit. Neither the patient nor her mother recollected any tick bites. After the diagnosis of Lyme borreliosis presenting as multiple EMs following dissemination of the infection, treatment with azithromycin $(10 \mathrm{mg} / \mathrm{kg})$ was initiated. The antibiotic was administered twice on the first treatment day and once daily until day five. The EMs started to fade during the second treatment day and had disappeared by day four. Serology later confirmed the diagnosis of Lyme borreliosis but did not show a seroconversion towards spotted fever group Rickettsia (data not shown). Throughout the disease course, no symptoms indicating a rickettsial co-infection were noticed. At checkups two weeks and two months after the initial visit, the skin was clear and the patient was fully recovered.

\section{Discussion}

Derived from the Borrelia spp. and Rickettsia spp. infection rates in ticks of $17.7 \%$ and $7.9 \%$, respectively, a theoretical co-infection rate of $1.4 \%$ was calculated (infection rate of Borrelia spp. $\mathrm{x}$ infection rate of Rickettsia spp.). The measured co-infection rate in the 1273 I. ricinus that were tested for both bacteria was $1.4 \%$. The theoretical and actual co-infection rates in ticks were also calculated for the combinations of the commonest Borrelia genospecies (Borrelia garinii and B. afzelii) and Rickettsia species ( $R$. helvetica and $R$. monacensis) and theoretical and actual co-infection rates for these combinations were also nearly identical (data not shown). The detection of $R$. raoultii in I. ricinus is surprising as this rickettsial species is associated with Dermacentor spp. The $R$. raoultii prevalence in this study was only $0.08 \%$ and detection of this DNA might be attributed to a recent infected blood meal or a contamination of the tick sample with material from Dermacentor marginatus ticks, which had been collected simultaneously.

The measured co-infection rate of $1.4 \%$ in ticks indicates that this percentage of EM patients has also been exposed to Rickettsia spp.. In our study population of 67 EM cases, this would translate to one patient (calculated: $0.94 \mathrm{pa}-$ tients). Indeed one skin sample was found Rickettsia spp. positive. Although the study population is limited, this indicates that transmission of Rickettsia spp. can occur alongside of Borrelia spp. if the tick carries a double infection. The current study population was too small to draw a definite conclusion on the virulence and pathogenicity differences of different rickettsial species found in $I$. ricinus. However, as $R$. helvetica was almost four times more common in ticks than $R$. monacensis, by chance, $R$. helvetica would have been more likely to cause co-infections in EM. Both, $R$. helvetica and $R$. monacensis, are thought to be pathogenic but the full clinical picture or the virulence are not known for either of these two species $[11,12]$. In the current case, the patient did not seem to suffer any symptoms associated with a Rickettsia spp. infection. The erythematous lesions were attributed to the B. burgdorferi s.l. infection and the patient recovered completely after a five-day course of antibiotics. Average incubation time in children with multiple EM has been reported to lie between 10 and 25 days [13,14]. The rickettsial bacteria in the skin lesions have thus survived in the skin for a similarly long period without being eliminated by the patient's immune system. Future case reports and epidemiological studies would be necessary to evaluate the clinical spectra and virulence of $R$. helvetica and R. monacenis.

\section{Conclusions}

In conclusion, the co-infection rates in EM patients with Rickettsia spp. was as high as predicted by tick co-infection rates. All symptoms of the patient with $R$. monacensis coinfection can be explained by the B. afzelii infection and the virulence of this rickettsial agent remains unclear. 
Nevertheless, this study highlights the importance of considering co-infections when treating Lyme borreliosis.

\section{Consent}

Informed written consent for publication was obtained from the parents of the patient.

\section{Competing interests}

The authors declare that they have no competing interests.

\section{Authors' contributions}

NP and HS designed the study and NP collected samples and patient data. ETK carried out laboratory experiments and conducted data analysis. All authors contributed to the manuscript and approved the final version of the manuscript.

\section{Acknowledgements}

We would like to thank Manoj Fonville and Paul Hengeveld (both RIVM) for their excellent technical support and all contributing hospitals and physicians for collecting EM samples. This study was financially supported by the Ministry of Health, Welfare and Sport and performed under the frame of EurNegVec Cost Action TD1303.

\section{Author details}

${ }^{1}$ Centre for Infectious Disease Control Netherlands, National Institute for Public Health and Environment (RIVM), Bilthoven, The Netherlands. 2Department of Infectious Diseases, General Hospital "Dr. Josip Benčević", Slavonski Brod, Croatia.

Received: 29 August 2013 Accepted: 5 December 2013 Published: 10 December 2013

\section{References}

1. Mullegger RR, Glatz M: Skin manifestations of lyme borreliosis: diagnosis and management. Am J Clin Dermatol 2008, 9:355-368.

2. Piesman J, Dolan MC: Protection against lyme disease spirochete transmission provided by prompt removal of nymphal Ixodes scapularis (Acari: Ixodidae). J Med Entomol 2002, 39:509-512.

3. Sprong $H$, Wielinga PR, Fonville M, Reusken C, Brandenburg AH, Borgsteede F, Gaasenbeek C, van der Giessen JW: Ixodes ricinus ticks are reservoir hosts for Rickettsia helvetica and potentially carry flea-borne Rickettsia species. Parasit Vectors 2009, 2:41.

4. Tijsse-Klasen E, Fonville M, Reimerink JH, Spitzen-van der Sluijs A, Sprong H: Role of sand lizards in the ecology of Lyme and other tick-borne diseases in the Netherlands. Parasit Vectors 2010, 3:42.

5. Silaghi C, Gilles J, Hohle M, Pradel I, Just FT, Fingerle V, Kuchenhoff H, Pfister K: Prevalence of spotted fever group rickettsiae in Ixodes ricinus (Acari: Ixodidae) in southern Germany. J Med Entomol 2008, 45:948-955.

6. Nilsson K, Jaenson TG, Uhnoo I, Lindquist O, Pettersson B, Uhlen M, Friman G, Pahlson C: Characterization of a spotted fever group Rickettsia from Ixodes ricinus ticks in Sweden. J Clin Microbiol 1997, 35:243-247.

7. Parola P, Paddock CD, Raoult D: Tick-borne rickettsioses around the world: emerging diseases challenging old concepts. Clin Microbiol Rev 2005, 18:719-756.

8. Tijsse-Klasen E, Pandak N, Hengeveld P, Takumi K, Koopmans MP, Sprong H: Ability to cause erythema migrans differs between Borrelia burgdorferi sensu lato isolates. Parasit Vectors 2013, 6:23.

9. Christova I, Van De Pol J, Yazar S, Velo E, Schouls L: Identification of Borrelia burgdorferi sensu lato, Anaplasma and Ehrlichia species, and spotted fever group Rickettsiae in ticks from Southeastern Europe. Eur J Clin Microbiol Infect Dis 2003, 22:535-542.

10. Wieten RW, Hovius JW, Groen EJ, van der Wal AC, de Vries PJ, Beersma MF, Tijsse-Klasen E, Sprong H, Grobusch MP: Molecular diagnostics of Rickettsia africae infection in travelers returning from South Africa to The Netherlands. Vector Borne Zoonotic Dis 2011, 11:1541-1547.
11. Nilsson K: Septicaemia with Rickettsia helvetica in a patient with acute febrile illness, rash and myasthenia. J Infect 2009, 58:79-82.

12. Jado I, Oteo JA, Aldamiz M, Gil H, Escudero R, Ibarra V, Portu J, Portillo A, Lezaun MJ, Garcia-Amil C, et al: Rickettsia monacensis and human disease, Spain. Emerg Infect Dis 2007, 13:1405-1407.

13. Arnez M, Pleterski-Rigler D, Ahcan J, Ruzic-Sabljic E, Strle F: Demographic features, clinical characteristics and laboratory findings in children with multiple erythema migrans in Slovenia. Wien Klin Wochenschr 2001, 113:98-101.

14. Arnez M, Pleterski-Rigler D, Luznik-Bufon T, Ruzic-Sabljic E, Strle F: Children with multiple erythema migrans: are there any pre-treatment symptoms and/or signs suggestive for central nervous system involvement? Wien Klin Wochenschr 2002, 114:524-529.

doi:10.1186/1756-3305-6-347

Cite this article as: Tijsse-Klasen et al:: Co-infection of Borrelia burgdorferi sensu lato and Rickettsia species in ticks and in an erythema migrans patient. Parasites \& Vectors 2013 6:347.

\section{Submit your next manuscript to BioMed Central and take full advantage of:}

- Convenient online submission

- Thorough peer review

- No space constraints or color figure charges

- Immediate publication on acceptance

- Inclusion in PubMed, CAS, Scopus and Google Scholar

- Research which is freely available for redistribution 